\title{
Progress in anti-HER2 targeted therapy of metastatic lung cancer
}

\author{
Xian $\mathrm{Xiao}^{1}$ Yingjie $\mathrm{Jia}^{1 *}$ \\ ${ }^{1}$ First Teaching Hospital of Tianjin University of Traditional Chinese Medicine, National Clinical Research Center for Chinese Medicine \\ Acupuncture and Moxibustion
}

\begin{abstract}
Lung cancer is one of the most common malignant tumors in the world and one of the main causes of cancer death. In lung cancer, metastatic stage accounted for a large percentage. It is a global disease affecting human health, with high incidence rate, low malignant degree and other characteristics. After decades of exploration, anti-HER2 targeted therapy in breast has made breakthrough progress, obtained encouraging clinical efficacy, and fully improved the quality of the life of patients. Because of this, more and more researchers are focusing on anti-HER2 in metastatic lung cancer targeting research. For the past few years, new targeted drugs have been constantly developed for anti-HER2 in metastatic lung cancer, and promising data results have been obtained in clinical trials and cohort study. This article provides a review of the clinical research progress of anti-HER2 targeted therapy in metastatic lung cancer in recent years, with a view to further guiding clinical treatment and providing more treatment options for patients.
\end{abstract}

\section{Introduction}

Among all diagnosed tumors, lung cancer is the largest contributor with $18.4 \%$ mortality [1]. However, despite a number of new technologies coming out in lung cancer's diagnosis, identification and treatment, lung cancer is still characterized by high mortality rate. It is estimated that, in 2019, the 5-year survival rate for lung cancer is only $19 \%$, which is in sharp contrast to other causes of cancer death in the United States [2]. Further, over the past decade, the 5-year overall survival rate for patients with metastatic non-small cell lung cancer is less than $5 \%$ [3].

Because of the characteristics of poor prognosis and limited treatment on lung cancer, intensive and continued clinical research of new combination and therapies are still called for. In the last 20 years, there have been a variety of anti-Human Epidermal Growth Factor Receptor 2 (anti-HER2) agents used to treat breast cancer stepping into clinical practice [4]. The anti-HER2 treatment for early or metastatic HER-2 positive breast cancer, which achieves notable achievements, can led to a positive increasement in disease-free survival (DFS) and overall survival (OS). [5] A growing number of patients potentially benefit from the HER2-targeted therapies which have become an indispensable method in present breast cancer treatment. Besides, HER2-targeted therapies also develop in other tumors such as gastric, colon or biliary malignant cancer [6]. Regarding lung cancer, HER-2 mutations and HER-2 amplification have been considered as carcinogenic factors and potential therapeutic targets [7]. Despite that the mechanism of using HER-2 targeting drugs as monotherapy or combining it with chemotherapy are poorly understood [6], it is worth noting that the HER2-targeted antibody-drug ado-trastuzumab emtansine (TDM-1) shows an activity signal in the treatment with HER2-overexpressing (IHC 3+) metastatic Non-Small Cell Lung Cancer (NSCLC) [8]. For the last few years, an increasing number of clinical trials of anti-HER2 targeted therapy in metastatic lung cancer have emerged. This may open up new possibilities for future metastatic lung cancer treatment.

Therefore, in this review, we will focus on anti-HER2 targeted therapy of metastatic lung cancer in recent years, especially the mechanism and clinical data of different treatments, to offer more therapeutic schedule for clinical treatment.

\section{Mechanism of anti-HER2 targeted therapy}

Epidermal growth factor receptor 2(HER2), also known as ERBB2, is one of the EGFR family members of receptor tyrosine kinases. Basic experimental studies have confirmed the expression of HER2 in various cell lines including multiple adenocarcinoma and squamous cell carcinoma cell lines [9] The binding of ligand and HER3/HER4 leads to the formation of catalytically active homo and heterodimers, thus activating several downstream pathways, including PI3K/AKT/mTOR and MEK/ERK, participating in the progress of cellular proliferation, differentiation, migration and apoptosis [10-13]. Despite there is no clear direct ligand for HER2., in fact, it is all other ERBB families' preferred dimerization bonder [13-14]. In addition, HER2 has a unique basal tyrosine kinase activity. Consequently, HER2 plays a significant role in signal transduction and when it loses, its function tumor will progress. The

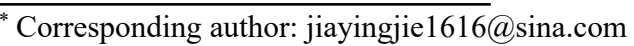


alterations of HER2 has been recognized as a driving factor in the development and progression of lung cancer and a potential therapeutic target. HER2 gene mutations, HER2 gene amplification, HER2 protein overexpression are three main forms of HER2 alteration which have been proven [15]. HER2 mutations are reported to happen in up to $4 \%$ of NSCLC patients and the most commonly encountered mutation is in-frame insertions in exon 20 [16-17]. HER2 gene amplification is observed in approximately $10-20 \%$ of NCSLC patients, while the proportion of HER2 protein overexpression in NSCLC is about 30\%-50\% according to the statistics [18]. There are different targeted drug therapies for different HER2 alterations, and they are summarized as follows.

\section{Trastuzumab}

Trastuzumab is a monoclonal antibody against HER2, which has been shown to be effective in the treatment of HER2-positive breast cancer. In recent years, with the continuous application of targeted therapy in the field of lung cancer treatment, trastuzumab, a the brief Her2-targeted drug, was on of the first used in clinical trials to treat patients with HER2-altered NSCLC.Studies have confirmed that trastuzumab plays a role mainly through promoting HER2 receptor down-regulation and degradation. Meanwhile, trastuzumab also can activate antibody-dependent cellular cytotoxicity to achieve the purpose of preventing the tumor's proliferation, differentiation, migration and apoptosis [19]. For now, preclinical data have demonstrated that trastuzumab is effective in NSCLC. However, the vast majority of trastuzumab clinical trial have not shown a demonstrable advantage for the NSCLC patients as monotherapy or combined with chemo-therapy [20]. In spite of this, there are still some clinical records showing trastuzumab to be effective, which suggests a potential and new direction.

The European EUHER2 cohort study identified 101 eligible metastatic NSCLC patients with a known HER2 exon-20 insertion, from 38 centers, and five-seventh of them received trastuzumab treatment. Subset analysis revealed that five-seventh trastuzumab-treated patients had a higher overall response rate (ORR) of $50.9 \%$ compared with conventional chemotherapy-treated patients, with an ORR of $43.5 \%$ [21]. This study shows the promise of trastuzumab HER2-targeted therapy in metastatic HER2-mutation NSCLC.

Corey J. Langer et al. reported a randomized phase II in patients with metastatic NSCLC that were positive for HER-2/neu by immunohistochemistry $1+$ to $3+$, evaluating the curative effect of combination carboplatin, paclitaxel, and trastuzumab [22]. Final results showed early acticivity with a median survival of 10.1 months (1-year survival rate , $42 \%$ ). In terms of toxic side effects, anemia has primary interim toxicity, and neutropenia has the most primary interim toxicity. Other toxicological reactions were not soginificantly different from those of carboplatin and paclitaxel alone. Thus, the cytotoxicity of the combination therapy is not worse than that of chemotherapy alone. Combination treatment is feasible.
At the same time, several seminal cases of HER2-positive NSCLC trearted with trastuzumab combined with chemotherapy have been constantly reported. Cappuzzo F. et al. treated a metastatic lung adenocarcinoma patient who was detected a HER2 exon 20 mutation with weekly trastuzumab and paclitaxel. After a four-month therapy, the level of carcinoembryonic antigen (CEA) went from 44 ng per milliliter to $1.4 \mathrm{ng}$ per milliliter, and chest $\mathrm{CT}$ showed almost complete disappearance of lung metastases [20].

Although there have also been some clinical trials with negative results [23-24], trastuzumab combined with chemotherapy is still potentially beneficial. The efficacy of trastuzumab combined with chemotherapy in the treatment of patients with HER-2 positive NSCLC still needs to be demonstrated by further muli-center,large-sample prospective or cohort studies to prove its efficacy and safety. Trastuzumab in combined with chemotherapy may offer new hope for patients who have no other treatment options.

\section{Pyrotinib}

Pyrotinib is a new targeted drug for anti-HER2 therapy, which has achieved good efficacy in clinical trials in recent years. Pyrotinib is an oral drug, the irreversible pan-HER receptor tyrosine kinase inhibitor (TKI), which can target effectively against epidermal growth factor receptor (EGFR)/HER1 and HER2 [25]. Phase I study and biomarker analysis of pyrotinib showed that the inhibition of pyrotinib on multiple HER receptors was irreversible, and the proliferation of HER2-overexpressed cells was effectively inhibited by pyrotinib in vitro and in vivo [26-27]. The combination of pyrotinib and capecitabine has shown good results in the treatment of HER2-positive metastatic breast cancer and has been approved for use in China. For metastatic lung cancer, pyrotinib has shown great potential, and has attracted the attention of many clinical researchers. Recent studies have indicated that pyrotinib mainly shows positive effects in HER2 mutation lung cancer patients. Reports are as follows.

In 2019, Y. Wang et al. reported for the first time the efficacy and safety of pytotinib in the treatment of HER2 mutated NSCLC [25]. In Y. Wang's vitro research, compared with afatinib and TDM-1, pyrotinib had a more obvious inhibitory effect on tumor growth. Moreover, the data showed that pHER2, pERK and pAkt were strongly inhibited by pyrotinib. A phase II clinical trial enrolling 15 patients with HER2-mutant NSCLC, found that pyrotinib led to median PFS of 6.4 months with an objective response rate (ORR)of 53.3\%.

Caicun Zhou et al. reported a multicenter, open-Label, single-Arm, phase II study on pyrotinib in HER2-mutant metastatic lung adenocarcinoma after platinum-based chemotherapy [25]. They enrolled 60 patients who had different types of Her2 mutations, and 44 (73.3\%) patients were detected with 12-bp exon 20.

Insertion.The results showed that pyrotinib had strong anti-tumor activity and low toxicity against different types of HER2 mutation patients. IRC-assessed 
ORR was 30.0\%. The 12-bp exon 20 Her2-mution patient objective response rate was $27.3 \%$. At the same time, the data suggest that pyrotinib is not less effective in patients with brain metastases. Meanwhile, the incidence of treatment-related grade 3 or 4 adverse reactions was $28.3 \%$, among which diarrhea was the most common. Thus, it can be seen that pyrotinib has good anti-tumor efficacy and high safety in patients with HER2 mutation NSCLC during chemotherapy.

\section{T-DM1(Trastuzumab-Emtansin)}

Trastuzumab emtansine(also known as T-DM1) is a HER2-targeted antibody-drug combination that connects trastuzumab with the anti-microtubule drug emtansine [28].T-DM1 can effectively target and deliver DM1 to HER2-positive cancer cells, maximizing the therapeutic index of DM1 and effectively reducing the off-target effect. T-DM1 has been approved for the treatment of advanced breast cancer patients with HER2 amplification and overexpression [8]. In preclinical studies, T-DM1 is demonstrated available in vitro growth suppression of HER2-mutation NSCLC cells [29]. Meanwhile, the vitro animal experimental data showed that T-DM1 inhibited the growth of SBC-3/SN-38 xenografts more obviously compared with trastuzumab, showing better potential against small cell lung cancer (SCLC) [30]. T-DM1 has also shown vital antitumor effects in clinical trials.

A multicenter, single-arm clinical trial enrolled 49 patients with metastatic NSCLC with HER 2 overexpression (29 IHC 2+, 20 IHC 3+) [8]. No significant treatment response was observed in the IHC2 + cohort. However, there were four partial responses observed in the IHC $3+$ cohort with an ORR of , $20 \%(95 \% \mathrm{CI}, 5.7 \%$ to $3.7 \%)$. In the IHC $2+$ and $3+$ cohorts, clinical benefit rates were $7 \%$ and $30 \%$, respectively. The results revealed that the T-DM1 showed significant anti-tumor activity in metastatic NSCLC with HER2-overexpressing (IHC3+). Due to the limitations of the trial, the data cannot demonstrate that T-DM1 was absolutely effective against HER2-overexpressing metastatic NSCLC. Further basic and clinical experiments are needed to identify changes in the HER2 pathway to further optimize the adaptation of TDM1 in NSCLC populations.

A phase basket II trial researched NSCLC patients with HER2 overexpression, application, or mutation treated with T-DM1[28]. Among the 15 enrolled patients who met the criteria, there were 5 IHC $3+, 3$ IHC 2+/FISH-positive, 7 with HER2 mutations NSCLC. All enrolled patients received $3.6 \mathrm{mg} / \mathrm{kg}$ of T-DM1 intravenously every 3 weeks until disease progression. The final data showed a partial response rate of $44 \%$ in patients with HER2 mutations. (95\% CI, 22\% to $69 \%$ ), with a median PFS of 5 months (95\% CI, 3 to 9 months). Collectively, T-DM1 has a good anti-tumor effect in HER2-mutant lung cancers. Further study on the T-DM1 is warranted. Based on this clinical trial, the NCCN has included T-DM1 in its Clinical Practice Guidelines for the treatment of metastatic HER2-mutation NSCLC patients.
However, what deserves our attention is T-DM1, which also has related side effects in clinical application. In a clinical trial, thrombocytopenia occurred in $40 \%$ of the enrolled patients. Thus, platelet count monitoring is a necessary measure during the use of T-DM1.Beyond that, T-DM1 can also affect liver metabolism, kidney metabolism, gastrointestinal function, etc. [31]

T-DM1 has initially shown clinical value in lung cancer, but it is worth noting that the related toxic and side effects in clinical application deserve comprehensive management. It is hoped that there will be more clinical trials of T-DM1 and greater breakthroughs in the treatment of lung cancer.

\section{Trastuzumab deruxtecan (DS8201)}

Trastuzumab deruxtecan (known as T-DXd; DS-8201a) is a HER2-directed antibody and DNA topoisomerase I inhibitor conjugate. Basic experimental studies have shown that DS8201 has about twice the drug-antibody ratio of T-DM1 [32]. At present, DS8201 is used for the treatment of a variety of HER2-expressing tumors, including HER2-expressing metastatic colorectal cancer, HER2-positive metastatic breast cancer and so on [33]. Recent studies have shown encouraging efficacy of DS8201 in the treatment of NSCLC. In the 2021 NCCN Clinical Practice Guidelines for NSCLC, DS8201 was first recommended for the treatment of HER2 mutant NSCLC.

Recently, an ongoing phase II clinical cohort study of DS8201 has yielded encouraging interim results. DESTINY-Lung01 is a cohort study evaluating the anti-tumor efficacy of DS8201 in HER2 overexpression and HER 2 mutation NSCLC. A total of 65 patients with HER2 mutation and 49 patients with HER2 overexpression NSCLC were enrolled in the cohort. Among the HER2 overexpression NSCLC patients, the statistical found the ORR of $24.5 \% \quad(95 \%$ CI:13.3\%-38.9\%), with a e disease control rate (DCR) was $69.4 \%$ (95\% CI:54.6\%-81.8\%). All patients had at least one treatment acute adverse event, mainly gastrointestinal adverse reaction $(59.2 \%)$. The ORR in patients with the HER2 mutation was $61.9 \%(95 \%$ CI:45.6\%-76.4\%), and the DCR was $90.5 \%$ (95\% CI: $77.4 \%-97.3 \%$ ). Third -level adverse drug reactions occurred in $64 \%$ of the patients, including neutrophils decline (26.2\%) and anemia (16.7\%) [34-35].

At present, there are a few results of cohort studies on DS8201, but it can be seen from current results that DS8201 initially shows anti-tumor activity. However, interstitial pneumonia may be a more serious side effect of DS8201, where prompt detection and intervention are required.

\section{Other}

In addition to the above drugs, there are a number of small-molecule anti-tumor agents targeting HER2 that have achieved initial efficacy in the treatment of HER2-mutated, HER2-amplified and HER2-overexpressed NSCLC. However, because of their 
limited efficacy, they have not been used in clinical practice.

Afatinib, is a non-reversible pan-HER inhibitor. Afatinib has shown positive efficacy and been approved to treat of patients with EGFR mutation in NSCLC. Basic research data showed that afatinib could restrain both HER2-amplified and HER2-mutant NSCLC cells through G1 arrest and apoptosis [36]. Li Zhang et al. enrolled 118 patients with stage IV/recurrent HER2 mutations and divided them into two cohorts to evaluate the association between different mutant subtypes and clinical outcomes. The results of cohort study showed that HER2 mutated NSCLC had significant heterogeneity, and different HER2 mutated subtypes have different sensitivity to HER2 therapy. Among them, patients with G778_P780DUP and G776DELINSVC mutant subtypes continued to benefit from afatinib therapy [37]. However, up to now, except for a few cases, most clinical studies on afatinib have had negative results. The OS, ORR and DFS of afatinib in the treatment of HER2 abnormal NSCLC are not ideal. $\mathrm{NCCN}$ was also removed as a recommended drug for the treatment of HER2 mutated NSCLC due to its poor efficacy and insufficient clinical trials.

Poziotinib is a novel targeted drug targeting rare EGFR and HER2 exon 20 insertion mutations. The ESMO 2020 Annual Meeting presented the results of a cohort study of poziotinib in patients with HER2-20 exon insertion mutation (ZENITH20-2). Among the 74 evaluable patients enrolled, the statistical data showed that the ORR and DCR were $35.1 \%$ and $70 \%$, and responsive diazepam was observed in most subgroups. However, compared with DS8201, poziotinib has poor efficacy and tolerability, and more patients need to reduce or discontinue medication due to adverse reaction. In the ZENITH20-2 cohort study, $88 \%$ of the patients were suspended, and $68 \%$ of the patients were reduced, and $10 \%$ of patients were permanently discontinued, which was regrettable. The FBA therefore did not approve it listing.

Neratinib (known as HKI-272), is an oral, non-reversible dual restrainer of EGFR and HER2 [38]. At the World Conference on Lung Cancer 2020, two cohort studies were reported in patients with metastatic HER2 mutated NSCLC treated with neratinib + tamrolimus or neratinib + trastuzumab. Results showed that neratinib as a monotherapy had limited activity in HER2-mutated NSCLC, and that neratinib combined with tamrolimus or trastuzumab produced greater efficacy and long-lasting remission in patients with HER2-mutated NSCLC.

Dacomitinib, developed by Pfizer, is a pan-HER inhibitor that irreversibly binds to HER2, HER1 (EGFR), and HER4 tyrosine kinases [39]. It is also one of the first targeted drugs to start clinical trials against the HER2 variant of lung cancer. Data from a cohort study showed that dacomitinib did not respond well to NSCLC patients with specific HER2 exon 20 insertions [40]. The clinical series reported only 3 of 26 patients with HER2-activated mutations responded, and the ORR was only $12 \%$. None of the four patients with HER2 amplification achieved objective response. Meanwhile, the incidence of treatment-related toxicity was high, in which diarrhea accounted for $90 \%$.

\section{Conclusion}

HER2 has been identified as a driving factor in the development and progression of lung cancer. This article summarized the research progress of anti-HER2 in the treatment of metastatic lung cancer in recent years. These HER2-targeted clinical studies have made some breakthroughs, providing more options for patients and improving the quality of life, but clinical studies on HER2 generally have fewer patients enrolled, and clinical trial results need to be verified in larger multi-center clinical trials. HER2-targeted therapy also has different degrees of treatment-related toxic and side effects, and close monitoring of adverse reactions is needed in clinical treatment. With regard to these novel treatments, we expect that more mechanism-related studies will be conducted to find molecular markers that can predict their efficacy, thus further guiding clinical treatment. A multi-center clinical cohort study with a larger sample size is expected to provide new directions and possibilities for clinical anti-HER2 targeted therapy.

\section{References}

1. Bray F, Ferlay J, Soerjomataram I, Siegel RL, Torre LA, Jemal A. Global cancer statistics 2018: GLOBOCAN estimates of incidence and mortality worldwide for 36 cancers in 185 countries. CA Cancer J Clin. 68(6):394-424(2018)

2. Nasim F, Sabath BF, Eapen GA. Lung Cancer. Med Clin North Am. 103(3):463-73(2019)

3. Arbour KC, Riely GJ. Systemic Therapy for Locally Advanced and Metastatic Non-Small Cell Lung Cancer: A Review. JAMA. 322(8):764-74(2019)

4. Yao M, Fu P. Advances in anti-HER2 therapy in metastatic breast cancer. Chin Clin Oncol. 7(3):27(2018)

5. Loibl S, Gianni L. HER2-positive breast cancer. Lancet. 389(10087):2415-29(2017)

6. Da Silva J, Jouida A, Ancel J, Dalstein V, Routhier J, Delepine G, et al. FHIT (low) /pHER2(high) signature in non-small cell lung cancer is predictive of anti-HER2 molecule efficacy. J Pathol. 251(2):187-99(2020)

7. Li BT, Ross DS, Aisner DL, Chaft JE, Hsu M, Kako SL, et al. HER2 Amplification and HER2 Mutation Are Distinct Molecular Targets in Lung Cancers. J Thorac Oncol. 11(3):414-9(2016)

8. Peters S, Stahel R, Bubendorf L, Bonomi P, Villegas A, Kowalski DM, et al. Trastuzumab Emtansine (T-DM1) in Patients with Previously Treated HER2-Overexpressing Metastatic Non-Small Cell Lung Cancer: Efficacy, Safety, and Biomarkers. Clin Cancer Res. 25(1):64-72(2019)

9. Weiner DB, Nordberg J, Robinson R, Nowell PC, Gazdar A, Greene MI, et al. Expression of the neu 
gene-encoded protein (P185neu) in human non-small cell carcinomas of the lung. Cancer Res. 50(2):421-5(1990)

10. Daly JM, Jannot CB, Beerli RR, Graus-Porta D, Maurer FG, Hynes NE. Neu differentiation factor induces ErbB2 down-regulation and apoptosis of ErbB2-overexpressing breast tumor cells. Cancer Res. 57(17):3804-11(1997)

11. Graus-Porta D, Beerli RR, Hynes NE. Single-chain antibody-mediated intracellular retention of ErbB-2 impairs Neu differentiation factor and epidermal growth factor signaling. Mol Cell Biol. 15(3):1182-91(1995)

12. Riese DJ, 2nd, Stern DF. Specificity within the EGF family/ErbB receptor family signaling network. Bioessays. 20(1):41-8(1998)

13. Spector NL, Blackwell KL. Understanding the mechanisms behind trastuzumab therapy for human epidermal growth factor receptor 2-positive breast cancer. J Clin Oncol. 27(34):5838-47(2009)

14. Arcila ME, Chaft JE, Nafa K, Roy-Chowdhuri S, Lau C, Zaidinski M, et al. Prevalence, clinicopathologic associations, and molecular spectrum of ERBB2 (HER2) tyrosine kinase mutations in lung adenocarcinomas. Clin Cancer Res. 18(18):4910-8(2012)

15. Peters S, Zimmermann S. Targeted therapy in NSCLC driven by HER2 insertions. Transl Lung Cancer Res. 3(2):84-8(2014)

16. Shigematsu H, Takahashi T, Nomura M, Majmudar $\mathrm{K}$, Suzuki M, Lee H, et al. Somatic mutations of the HER2 kinase domain in lung adenocarcinomas. Cancer Res. 65(5):1642-6(2005).

17. Buttitta F, Barassi F, Fresu G, Felicioni L, Chella A, Paolizzi D, et al. Mutational analysis of the HER2 gene in lung tumors from Caucasian patients: mutations are mainly present in adenocarcinomas with bronchioloalveolar features. Int $\mathrm{J}$ Cancer. 119(11):2586-91(2006)

18. Mazieres J, Peters S, Lepage B, Cortot AB, Barlesi $\mathrm{F}$, Beau-Faller $\mathrm{M}$, et al. Lung cancer that harbors an HER2 mutation: epidemiologic characteristics and therapeutic perspectives. J Clin Oncol. 31(16):1997-2003(2013)

19. Molina MA, Codony-Servat J, Albanell J, Rojo F, Arribas J, Baselga J. Trastuzumab (herceptin), a humanized anti-Her2 receptor monoclonal antibody, inhibits basal and activated Her2 ectodomain cleavage in breast cancer cells. Cancer Res. 61(12):4744-9(2001)

20. Cappuzzo F, Bemis L, Varella-Garcia M. HER2 mutation and response to trastuzumab therapy in non-small-cell lung cancer. $\mathrm{N}$ Engl $\mathrm{J}$ Med. 354(24):2619-(2006)

21. Mazieres J, Barlesi F, Filleron T, Besse B, Monnet I, Beau-Faller $M$, et al. Lung cancer patients with HER2 mutations treated with chemotherapy and
HER2-targeted drugs: results from the European EUHER2 cohort. Ann Oncol. 27(2):281-6(2016)

22. Langer CJ, Stephenson P, Thor A, Vangel M, Johnson DH, Eastern Cooperative Oncology Group S. Trastuzumab in the treatment of advanced non-small-cell lung cancer: is there a role? Focus on Eastern Cooperative Oncology Group study 2598. J Clin Oncol.22(7):1180-7(2004)

23. Gatzemeier U, Groth G, Butts C, Van Zandwijk N, Shepherd F, Ardizzoni A, et al. Randomized phase II trial of gemcitabine-cisplatin with or without trastuzumab in HER2-positive non-small-cell lung cancer. Ann Oncol. 15(1):19-27(2004)

24. Lara PN, Jr., Laptalo L, Longmate J, Lau DH, Gandour-Edwards R, Gumerlock PH, et al. Trastuzumab plus docetaxel in HER2/neu-positive non-small-cell lung cancer: a California Cancer Consortium screening and phase II trial. Clin Lung Cancer. 5(4):231-6(2004)

25. Wang Y, Jiang T, Qin Z, Jiang J, Wang Q, Yang S, et al. HER2 exon 20 insertions in non-small-cell lung cancer are sensitive to the irreversible pan-HER receptor tyrosine kinase inhibitor pyrotinib. Ann Oncol. 30(3):447-55(2019)

26. Li X, Yang C, Wan H, Zhang G, Feng J, Zhang L, et al. Discovery and development of pyrotinib: A novel irreversible EGFR/HER2 dual tyrosine kinase inhibitor with favorable safety profiles for the treatment of breast cancer. Eur J Pharm Sci. 110:51-61(2017)

27. Ma F, Li Q, Chen S, Zhu W, Fan Y, Wang J, et al. Phase I Study and Biomarker Analysis of Pyrotinib, a Novel Irreversible Pan-ErbB Receptor Tyrosine Kinase Inhibitor, in Patients With Human Epidermal Growth Factor Receptor 2-Positive Metastatic Breast Cancer. J Clin Oncol. 35(27):3105-12(2017)

28. Li BT, Shen R, Buonocore D, Olah ZT, Ni A, Ginsberg MS, et al. Ado-Trastuzumab Emtansine for Patients With HER2-Mutant Lung Cancers: Results From a Phase II Basket Trial. J Clin Oncol. 36(24):2532-7(2018)

29. Phillips GD, Fields CT, Li G, Dowbenko D, Schaefer G, Miller K, et al. Dual targeting of HER2-positive cancer with trastuzumab emtansine and pertuzumab: critical role for neuregulin blockade in antitumor response to combination therapy. Clin Cancer Res.20(2):456-68(2014)

30. Morimura O, Minami T, Kijima T, Koyama S, Otsuka T, Kinehara Y, et al. Trastuzumab emtansine suppresses the growth of HER2-positive small-cell lung cancer in preclinical models. Biochem Biophys Res Commun. 488(4):596-602(2017)

31. Hotta K, Aoe K, Kozuki T, Ohashi K, Ninomiya K, Ichihara E, et al. A Phase II Study of Trastuzumab Emtansine in HER2-Positive Non-Small Cell Lung Cancer. J Thorac Oncol. 13(2):273-9(2018)

32. Tsurutani J, Iwata H, Krop I, Janne PA, Doi $T$, Takahashi S, et al. Targeting HER2 with Trastuzumab Deruxtecan: A Dose-Expansion, Phase 
I Study in Multiple Advanced Solid Tumors. Cancer Discov. 10(5):688-701(2020)

33. Keam SJ. Trastuzumab Deruxtecan: First Approval. Drugs. 80(5):501-8(2020)

34. OA04,05-Trastuzumab Deruxtecan in HER2-Overexpressing Metastatic Non-Small Cell Lung Cancer:Interim Results of DESTINY-Lung01.Presented at :WCLC 2020 Virtual; (January 28-31, 2021)

35. MA11.03-Trastuzumab Deruxtecan in HER2-Mutated Metastatic Non-Small Cell Lung Cancer(NSCLC):Interim Results of DESTINY-Lung01.Presented at :WCLC 2020 Virtual; (January 28-31, 2021)

36. Suzawa K, Toyooka S, Sakaguchi M, Morita M, Yamamoto H, Tomida S, et al. Antitumor effect of afatinib, as a human epidermal growth factor receptor 2-targeted therapy, in lung cancers harboring HER2 oncogene alterations. Cancer Sci. 107(1):45-52(2016)
37. Fang W, Zhao S, Liang Y, Yang Y, Yang L, Dong X, et al. Mutation Variants and Co-Mutations as Genomic Modifiers of Response to Afatinib in HER2-Mutant Lung Adenocarcinoma. Oncologist. 25(3):e545-e54(2020)

38. Bose P, Ozer H. Neratinib: an oral, irreversible dual EGFR/HER2 inhibitor for breast and non-small cell lung cancer. Expert Opin Investig Drugs. 18(11):1735-51(2009)

39. Engelman JA, Zejnullahu K, Gale CM, Lifshits E, Gonzales AJ, Shimamura T, et al. PF00299804, an irreversible pan-ERBB inhibitor, is effective in lung cancer models with EGFR and ERBB2 mutations that are resistant to gefitinib. Cancer Res. 67(24):11924-32(2007)

40. Kris MG, Camidge DR, Giaccone G, Hida T, Li BT, O'Connell J, et al. Targeting HER2 aberrations as actionable drivers in lung cancers: phase II trial of the pan-HER tyrosine kinase inhibitor dacomitinib in patients with HER2-mutant or amplified tumors. Ann Oncol.26(7):1421-7(2015). 